June, 1993

\title{
SELFCONSISTENT DESCRIPTION OF A THERMAL PION GAS
}

\author{
R. RAPP and J. WAMBACH U \\ Institut für Kernphysik \\ Forschungszentrum Jülich \\ D-5170 Jülich, F.R.G
}

\begin{abstract}
We examine a hot pion gas by including medium modifications of the two-body scattering amplitude as well as mean-field effects selfconsistently. In contrast to earlier calculations, the in-medium T-matrix is rather close to the free one while the mean-field potential agrees well with lowest-order estimates. We also discuss the validity of the quasiparticle approximation. It is found that it is reliable for temperatures up to $\sim 150 \mathrm{MeV}$. Above this temperature off-shell effects in the pion selfenergy become important, especially if the pions are strongly out of chemical equilibrium.
\end{abstract}

PACS Indices: 13.75.Lb

\footnotetext{
${ }^{1}$ also: Department of Physics, University of Illinois at Urbana-Champaign, 1100 West Green St., Urbana, IL 61081, USA

and: Institut für theoretische Kernphysik, Universität Bonn, D-5300 Bonn, F.R.G.
} 


\section{Introduction}

In ultrarelativistic heavy ion collisions several hundred particles are produced in the final state. In the midrapidity region at CERN energies of $200 \mathrm{GeV} / \mathrm{A}$ most of them are pions (with a pion-to-baryon ratio of $\sim 6: 1$ ). The lifetime of the pionic fireball is a few $\mathrm{fm} / \mathrm{c}$ and the freeze-out volume is typically $10^{3} \mathrm{fm}^{3}$. The theoretical understanding of such a thermal 'pion gas' is currently of great interest in connection with possible signals from the quark-gluon plasma (QGP) phase transition [四]. On the other hand a hot and dense Bose gas is also interesting from a many-body point of view since one may expect interesting correlations associated with the statistics.

Several aspects of the thermal pion gas have been discussed previously, including meanfield effects [2] and medium modifications of the $\pi \pi$ cross section [3]. It is the purpose of the present paper to give a unified description of these processes by using a reliable model for the vacuum $\pi \pi$ interaction and requiring selfconstistency at the two-body level. This leads to a Brueckner scheme, familiar from the microscopic many-body theory of nuclear matter. Throughout the discussion we shall assume that the gas is in thermal but not necessarily in chemical equilibrium $\left(\mu_{\pi} \neq 0\right)$. The latter seems to be required from fits to pion $p_{T}$-spectra, which yield $\mu_{\pi} \sim m_{\pi}$ [4]. The assumption of thermal equilibrium, on the other hand, seems reasonable from the following simple estimate: at $200 \mathrm{GeV} / \mathrm{A}$ the freeze-out density $n_{\pi}$ of pions produced at midrapidity is $\sim 0.3 \mathrm{fm}^{-3}$. With $N_{\pi} \approx 400$ this yields a freeze-out radius $R_{f} \sim 7 \mathrm{fm}$. Taking an average $\pi \pi$ cross section $\langle\sigma\rangle=15 \mathrm{mb}$ gives a mean free path $\lambda=1 / n_{\pi}\langle\sigma\rangle \sim 2.2 \mathrm{fm}$ and hence the mean number of collisions is $\sim 3$. Thermalization is further corroborated in a scenario where initially a quark-gluon plasma $(Q G P)$ is formed. Here QCD string breaking models as well as parton cascade simulations also yield thermal equilibration which should survive during the hadronization. 


\section{The Vacuum $\pi \pi$ Interaction}

The starting point for our description of the hot pion gas is the vacuum $\pi \pi$ interaction model of ref. [5] which is based on meson exchange. Here the basic meson-meson interaction is constructed from an effective meson Lagrangian with phenomenological form factors at the vertices. From these vertices two-body pseudopotentials are constructed including the most important $s$ - and $t$-channel meson exchanges. Among these the $t$-channel $\rho$ exchange between $\pi \pi$ states and the $s$-channel $\rho$ pole term will be most important for our discussion. We employ the Blankenbecler-Sugar (BbS) reduction [6] of the 4-dimensional Bethe-Salpeter equation maintaining covariance [7]. The $\pi \pi T$-matrix for given angular momentum $J$ and isospin $I$ is obtained as

$$
\begin{aligned}
T_{\pi \pi}^{J I}\left(Z, q_{1}, q_{2}\right)= & V_{\pi \pi}^{J I}\left(Z, q_{1}, q_{2}\right)+ \\
& +\int_{0}^{\infty} d k k^{2} 4 \omega_{k}^{2} V_{\pi \pi}^{J I}\left(Z, q_{1}, k\right) G_{\pi \pi}^{0}(Z, k) T_{\pi \pi}^{J I}\left(Z, k, q_{2}\right),
\end{aligned}
$$

where $k=|\vec{k}|$ etc. ; $Z$ is the CMS energy and $G_{\pi \pi}^{0}(Z, k)$ the vacuum two-pion propagator in the CMS frame with pions of momenta $\vec{k}$ and $-\vec{k}$ (the Lohse et al. model also contains coupling to the $K \bar{K}$ channel which has been omitted for brevity in eq. (1) but which is included in the calculation). In the $\mathrm{BbS}$ form the two-pion propagator is given by

$$
G_{\pi \pi}^{0}(Z, k)=\frac{1}{\omega_{k}} \frac{1}{Z^{2}-4 \omega_{k}^{2}+i \eta}
$$

with $\omega_{k}^{2}=k^{2}+m_{\pi}^{2}$. This model gives a good description of the phase shifts and inelasticities up to $\sim 1.5 \mathrm{GeV}$ which is more than sufficient for our purposes.

\section{Selfconsistency}

The most obvious medium modification of the $\pi \pi$ scattering in the gas surrounding is a change in momentum weight of the intermediate two-pion propagator, first studied in ref. [3]. Identifying the CMS frame with the thermal reference frame one has

$$
G_{\pi \pi}\left(Z, k ; \mu_{\pi}, T\right)=\frac{1}{\omega_{k}} \frac{\left(1+2 f_{k}\left(\mu_{\pi}, T\right)\right)}{Z^{2}-4 \omega_{k}^{2}},
$$


where $f_{k}=\left(\exp \left[\left(\omega_{k}-\mu_{\pi}\right) / T\right]-1\right)^{-1}$ is the thermal Bose factor and $\mu_{\pi}$ the chemical potential. The identification of the CMS frame with the thermal frame simplifies our calculations considerably (allowing for a relative velocity between the two frames we find that it effectively acts like a change in $\mu_{\pi}$ which is a parameter for us anyway). At fixed $T$ the pion chemical potential fixes the density via

$$
n_{\pi}\left(\mu_{\pi}, T\right)=g_{\pi} \int \frac{d^{3} q}{(2 \pi)^{3}} f_{q}\left(\mu_{\pi}, T\right)
$$

where $g_{\pi}=3$ is the isospin degeneracy factor. We use a temperature range of 100-200 $\mathrm{MeV}$. A temperature of $100 \mathrm{MeV}$ is roughly the lowest temperature from thermal fits to $p_{T}$-spectra at the AGS. On the other hand $200 \mathrm{MeV}$ should be an upper limit for purely hadronic models since one expects the phase transition into a QGP around this value. While thermal equilibrium seems a reasonable assumption it is not clear whether chemical equilibration is reached during the evolution of the pion gas. Indeed, fits of the CERN $p_{T}$-spectra could be improved with $\mu_{\pi} \sim 125 \mathrm{MeV}$ 《4. Hence we discuss both cases $\mu_{\pi}=0$ and $\mu_{\pi}=125 \mathrm{MeV}$. At the same temperature the latter gives a higher pion density reaching a maximal density $n_{\pi} \sim 0.7 \mathrm{fm}^{-3}$ at $200 \mathrm{MeV}$.

There is a second effect which needs to be considered [2]. The $\pi \pi$ interaction introduces a pion selfenergy $\Sigma_{\pi}$ ('mean field') which changes the single-pion dispersion relation

$$
\omega_{k}^{2}=m_{\pi}^{2}+k^{2}+\Sigma_{\pi}\left(\omega_{k}, k ; \mu_{\pi}, T\right)
$$

as a function of density and temperature. In terms of the forward-scattering amplitude $M_{\pi \pi}, \Sigma_{\pi}$ is expressed as [2, 8]:

$$
\Sigma_{\pi}\left(\omega, k ; \mu_{\pi}, T\right)=\int \frac{d^{3} p}{(2 \pi)^{3}} \frac{1}{2 \omega_{p}} f_{p}\left(\mu_{\pi}, T\right) M_{\pi \pi}\left(k^{(4)}, p^{(4)}\right) .
$$

Relating the forward-scattering amplitude to the T-Matrix as

$$
M_{\pi \pi}\left(E_{c m s}\right)=(2 \pi)^{3} E_{c m s}^{2} T_{\pi \pi}\left(E_{c m s}\right)
$$

where $E_{c m s}=\sqrt{s}=\left[(p+k)^{\mu}(p+k)_{\mu}\right]^{1 / 2}$ is the CMS energy of the two colliding pions, 
one can transform the selfenergy expression into

$$
\Sigma_{\pi}\left(\omega, k ; \mu_{\pi}, T\right)=\frac{\pi}{k} \int_{0}^{\infty} d p \frac{p}{\omega_{p}} f_{p}\left(\mu_{\pi}, T\right) \int_{E_{\min }}^{E_{\max }} d E_{c m s} E_{c m s}^{3} T_{\pi \pi}\left(E_{c m s}\right) .
$$

Here we have restricted ourselves to the on-shell T-Matrix neglecting the dependence on the total momentum $\vec{P}=\vec{k}+\vec{p}$ of the pair. Thus the energy integration bounds are given as

$$
E_{\max / \min }=\left(\omega^{2}+\omega_{p}^{2}+2 \omega \omega_{p}-k^{2}-p^{2} \pm 2 k p\right)^{1 / 2}
$$

For the forward-scattering T-Matrix we take the spin-isospin weighted sum including partial waves up to $J=2$ :

$$
T_{\pi \pi}\left(E_{c m s}\right)=\frac{1}{4 \pi} \sum_{I, J=0}^{2} \frac{(2 I+1)}{3}(2 J+1) T_{\pi \pi}^{J I}\left(E_{c m s}\right)
$$

which saturates the cross section in the relevant energy range.

It should be noted that the rate $\Gamma_{k}=-2 \operatorname{Im} \Sigma_{\pi} /\left(2\left(k^{2}+m_{\pi}^{2}\right)^{1 / 2}\right)$ deduced from eq. (8) is not entirely consistent with that obtained from the collision term of the bosonic Boltzmann equation. A correct account of the Bose correlations of the two interacting pions will modify the occupancy factor in (8) [9]. It turns out, however, that this is a small effect [10.

It is now evident that the pion selfenergy and the T-Matrix should be combined in a selfconsistent Brueckner scheme as indicated in Fig. 1. This implies that the selfenergy (8) is to be calculated from the in-medium T-Matrix which, on the other hand, should be obtained from the in-medium $2 \pi$ propagator including the pion selfenergy:

$$
G_{\pi \pi}\left(Z, k ; \mu_{\pi}, T\right)=\left(1+2 f_{k}\left(\mu_{\pi}, T\right)\right) \int \frac{i d \omega}{2 \pi} D_{\pi}(\omega, k) D_{\pi}(Z-\omega, k)
$$

where

$$
D_{\pi}(\omega, k)=\left[\omega^{2}-m_{\pi}^{2}-k^{2}-\Sigma_{\pi}\left(\omega, k ; \mu_{\pi}, T\right)\right]^{-1} .
$$

Together with the in-medium scattering equation,

$$
T_{\pi \pi}\left(\mu_{\pi}, T\right)=V_{\pi \pi}+V_{\pi \pi} G_{\pi \pi}\left(\mu_{\pi}, T\right) T_{\pi \pi}\left(\mu_{\pi}, T\right)
$$


equations (8), (11) and (12) define the selfconsistency problem (see also Fig. 1). It should be noticed that the pseudopotential $V_{\pi \pi}$ remains unchanged.

In the following we will discuss two different methods of calculating $G_{\pi \pi}$.

\section{Quasiparticle Approximation}

The quasiparticle approximation (QPA) is valid if the lifetime is long or more precisely if the quasiparticle energy

$$
e_{k} \equiv\left(m_{\pi}^{2}+k^{2}+R e \Sigma_{\pi}\left(e_{k}, k\right)\right)^{1 / 2}
$$

is much larger than its decay width $\Gamma_{k}$. In this case the energy-dependence of $R e \Sigma_{\pi}$ is expanded to first order around the 'quasiparticle pole' $e_{k}$ as

$$
\operatorname{Re} \Sigma_{\pi}(\omega, k) \approx \operatorname{Re} \Sigma_{\pi}\left(e_{k}, k\right)+\left.\frac{\partial \operatorname{Re} \Sigma_{\pi}(\omega, k)}{\partial \omega^{2}}\right|_{e_{k}}\left(\omega^{2}-e_{k}^{2}\right)
$$

One can then perform the folding integral (11) analytically which gives

$$
G_{\pi \pi}\left(Z, k ; \mu_{\pi}, T\right)=\frac{1}{\bar{\omega}_{k}} \frac{z_{k}^{2}\left(1+2 f_{k}\left(\mu_{\pi}, T\right)\right)}{Z^{2}-4 \bar{\omega}_{k}^{2}}
$$

with

$$
\begin{aligned}
z_{k} & \equiv\left(1-\left.\frac{\partial \operatorname{Re} \Sigma_{\pi}(\omega, k)}{\partial \omega^{2}}\right|_{e_{k}}\right)^{-1} \text { the polestrength } \\
\bar{\omega}_{k}^{2} & \equiv e_{k}^{2}+i z_{k} \operatorname{Im} \Sigma_{\pi}\left(e_{k}, k\right) \quad \text { quasipion dispersion relation }
\end{aligned}
$$

Together with this approximation eqs. (8),(11) and (13) are solved by iteration starting from the free pion dispersion relation and the vacuum T-Matrix. We keep the pion density $n_{\pi}$ fixed during the iteration by readjusting $\mu_{\pi}$ in each step (the final $\mu_{\pi}$ differs from the starting value $\mu_{\pi}^{(0)}$ by a small amount). The selfconsistent results are shown in Fig. 2. Already for chemical equilibrium $\left(\mu_{\pi}^{(0)}=0\right)$ we find a considerable reduction of the peak values in $I m T^{J I}$ as compared to the vacuum case in both s- and p-wave. This is in

agreement with the lowest-order results from refs. [3], 11]. The reduction is mainly caused 
by the Bose factors $(1+2 f)$ leading to a stronger weighting of the lower pion energies. For the same reason the near threshold region shows an enhancement over the vacuum T-Matrix especially in the s-wave. We define the pion 'optical potential' as [2]

$$
V_{\pi}(k) \equiv \frac{\Sigma_{\pi}\left(e_{k}, k\right)}{2\left(k^{2}+m_{\pi}^{2}\right)^{1 / 2}}
$$

which is shown in the lower part of Fig. 2. In agreement with refs. 20, 8] we find attraction in $R e V_{\pi}$ for low momenta. The selfconsistent potential, however, is significantly weaker than in lowest order. The maximum in $\operatorname{Im} V_{\pi}$ is due to formation of the $\rho$-resonance, whereas the non-zero values at $k=0$ arise from s-wave interaction with thermally moving pions.

\section{Off-shell Integration of $G_{\pi \pi}$}

To check the reliability of the QPA we perform the same calculations as described in the previous section, but the quasiparticle two-pion propagator is now replaced by a numerical integration of (11) accounting for the full off-shell properties of $\Sigma_{\pi}(\omega, k)$. Using the symmetry relation

$$
\Sigma_{\pi}(-\omega, k)=\Sigma_{\pi}(\omega, k)
$$

the in-medium propagator (11) can be written as

$$
G_{\pi \pi}\left(Z, k ; \mu_{\pi}, T\right)=\left(1+2 f_{k}\left(\mu_{\pi}, T\right)\right) \int_{Z / 2}^{\infty} \frac{i d \omega}{\pi} D_{\pi}(\omega, k) D_{\pi}(|Z-\omega|, k) .
$$

Fig. 3 shows the selfconsistent results for a chemically equilibrated pion gas $\left(\mu_{\pi}^{(0)}=0\right)$. At $T=125 \mathrm{MeV}$ the results coincide with the QPA within a few percent. For $T \gtrsim 150 \mathrm{MeV}$ the deviations become larger: the T-Matrices in s- and p-wave are now enhanced compared to the vacuum curve for most of the energy range (e.g. the peak value of the $\rho$-resonance increases by $\approx 30 \%$ at $T=200 \mathrm{MeV}$ ). This clearly must be an off-shell effect. From the lower part of Fig. 3 one can conclude that a first-order expansion of $R e \Sigma_{\pi}$ around $e_{k}$ does not describe the energy dependence correctly; in addition $\Gamma_{k} / e_{k} \sim 0.6$ around 
$k=300 \mathrm{MeV} / \mathrm{c}$, i.e. the quasiparticle lifetime becomes very short and renders the QPA invalid. The potentials $V_{\pi}(k)$ are now very close to the lowest-order results. As compared to the QPA a considerable amount of attraction is restored in the low-momentum region of $\operatorname{ReV}_{\pi}(k)$. We also investigate a scenario with finite chemical potential $\mu_{\pi}^{(0)}=125 \mathrm{MeV}$

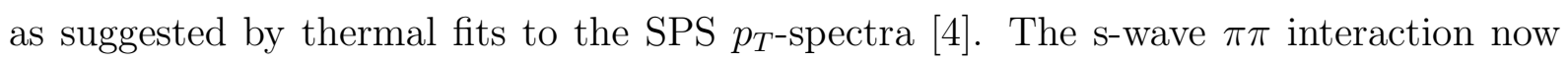
shows a strong accumulation of strength near threshold (Fig. 4) which might lead to quasibound $\pi^{+} \pi^{-}$pairs as suggested in ref. [11]. The latter possibility deserves further study. In the p-wave the changes of the $\rho$-resonance are appreciable: at highest temperatures the width decreases considerably which might be detectable via dilepton pairs coming from the midrapidity region.

\section{Summary}

Based on a selfconsistent Brueckner theory we have presented a numerical analysis of a hot, interacting pion gas in thermal equilibrium, which may be realized in future experiments at RHIC or LHC (at $\sqrt{s} \geq 100 \mathrm{GeV} / \mathrm{A}$ ). The selfconsistent Brueckner scheme accounts for statistical (Bose factors) as well as dynamical (selfenergy) modifications of the pion propagation in the gas. Using a realistic model for the vacuum $\pi \pi$ interaction we have solved the non-linear problem by iteration. We have compared results from the QPA to those taking full account of the off-shell behavior in the pion selfenergy. It is found that the QPA breaks down for temperatures $T \geq 150 \mathrm{MeV}$ and finite chemical potentials, i.e. high pion densities. In the full calculation we find a slight increase of the in-medium T-matrix - in contrast to refs. [3, 11], where only statistical modifications have been taken into account. The main cause of this enhancement is attributed to strong off-shell effects in the pion selfenergy. The single-particle potentials show considerable attraction for low pion momenta, although quantitatively somewhat less than our lowest-order results. As was shown recently by Koch and Bertsch [12], such an attraction is not able to explain the low- $p_{T}$ enhancement in the pion spectra of current experiments. However, as suggested 
by numerical simulations of the bosonic transport equation [13], an increase of the inmedium T-Matrix, as found in our calculations, is likely to ensure thermalization of the pionic fireball and hence to produce an excess of low- $p_{T}$ pions through the $(1+f)$ factors in the collision integral. This issue and the impact of finite baryon density in the pion gas will be addressed to a future publication.

\section{Acknowledgement}

We thank G. F. Bertsch, G. E. Brown, G. Chanfray, M. Prakash, E. V. Shuryak and P. Schuck for useful discussions. This work was supported in part by a grant from the National Science Foundation, NSF-PHY-89-21025. 


\section{References}

[1] J. Stachel and G. R. Young, Ann. Rev. Nucl. Part. Sci. 42 (1992) 537.

[2] E.V. Shuryak, Nucl. Phys. A533 (1991) 761.

[3] H.W. Barz, G.Bertsch, P.Danielewicz and H. Schulz, Phys. Lett. B275 (1992) 19.

[4] M. Kataja and P.V. Ruuskanen, Phys. Lett. B243 (1990) 181.

[5] D. Lohse, J.W. Durso, K. Holinde and J. Speth, Phys. Lett. B234 (1989) 235; Nucl. Phys. A516 (1990) 513.

[6] R. Blankenbecler and R. Sugar, Phys. Rev. 142 (1966) 1051.

[7] B. C. Pearce, K. Holinde and J. Speth, Nucl. Phys. A541 (1992) 663.

[8] A. Schenk, Nucl. Phys. B363 (1991) 97.

[9] G. Chanfray and D. Davesne, to be published.

[10] G. Chanfray, private communication.

[11] Z. Aouissat, G. Chanfray, P. Schuck and G. Welke, Zeit. Phys. A340 (1991) 347.

[12] V. Koch and G. Bertsch, Nucl. Phys. A522 (1992) 591.

[13] H.W. Barz, P. Danielewicz, H. Schulz and G. Welke, Phys. Lett. B287 (1992) 40. 


\section{FIGURE CAPTIONS}

Fig. 1 Set of selfconsistent equations for an interacting pion gas:

upper part: Dyson equation for in-medium pion propagation

lower part: in-medium $\pi \pi$ T-Matrix equation.

Fig. 2 Selfconsistent pion gas at $\mu_{\pi}^{(0)}=0$ in QPA:

upper part: imaginary part of the $\pi \pi$ T-Matrix in the $\mathrm{JI}=00$ - and $\mathrm{JI}=11$-channels for several temperatures (long-dashed lines: $T=125 \mathrm{MeV}$, short-dashed lines: $T=150$ MeV, dotted lines: $T=200 \mathrm{MeV}$ )

lower part: corresponding pion potentials (short-dashed lines: $T=150 \mathrm{MeV}$, dotted lines: $T=200 \mathrm{MeV}$; the dashed-dotted and dashed-double-dotted lines show the lowestorder results calculated with the vacuum T-Matrix for $T=150 \mathrm{MeV}$ and $T=200 \mathrm{MeV}$, respectively).

Fig. 3 Selfconsistent pion gas at $\mu_{\pi}^{(0)}=0$ with full off-shell integration of $G_{\pi \pi}(e q .(20))$ : upper part: imaginary part of the $\pi \pi$ T-Matrix (see Fig. 2)

lower part: real part of the pion potentials (see Fig. 2) and real part of the off-shell pion selfenergy at $T=200 \mathrm{MeV}$.

Fig. 4 Imaginary part of the selfconsistent $\pi \pi$ T-Matrix with full off-shell integration of $G_{\pi \pi}$ for $\mu_{\pi}^{(0)}=125 \mathrm{MeV}$ (long-dashed lines: $T=100 \mathrm{MeV}$, short-dashed lines: $T=150$ $\mathrm{MeV}$, dotted lines: $T=175 \mathrm{MeV}$, full lines: free space). 